
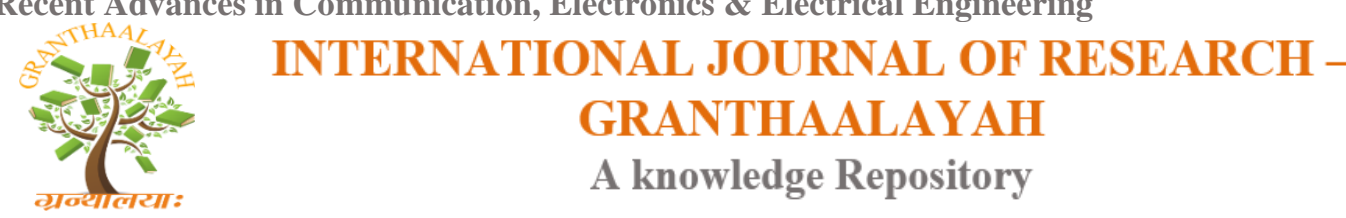

RACEEE - 17

\title{
DESIGN OF TRAFFIC ENGINEERED MULTI-PROTOCOL LABEL SWITCHING-TRANSPORT PROFILE (MPLS-TP) FOR THE ENHANCEMENT OF QUALITY OF SERVICE
}

\author{
Prabhavati Biradar ${ }^{* 1}$, Purushottama T $\mathbf{L}^{2}$ \\ ${ }^{* 1}$ M.Tech, Signal Processing, SIT Tumakuru, Karnataka - 572103, India \\ ${ }^{2}$ Assistant Professor, SIT Tumakuru, Karnataka - 572103, India
}

DOI: https://doi.org/10.29121/granthaalayah.v5.i4RACEEE.2017.3323

\begin{abstract}
Multiprotocol Label Switching (MPLS) is a switching technology. MPLS-TP is a progressive version of MPLS in the field of packet switched data network. MPLS-TP is a high-end technology which implements network methodologies in a transport layer. For managing virtual private network (VPN) services, MPLS is of developing enthusiasm to the service providers (SPs) and furthermore to VPN customers. MPLS-enabled routers allot a few labels to the packets, and in view of these allocated labels it produces sending decisions. The prime expectation after the MPLS technology is to eliminate the need of OSI model data link layered (layer-2) technology i.e. frame relay, Ethernet, asynchronous transfer mode (ATM). This paper gives data about the components of Multi-Protocol Label Switching (MPLS), correlation of MPLS with traditional Routing and Packet Switching, MPLS label format and MPLS operation.
\end{abstract}

Keywords: MPLS; MPLS Traffic Engineering; MPLS Virtual Private Network; Routing Protocols.

Cite This Article: Prabhavati Biradar, and Purushottama T L. (2017). "DESIGN OF TRAFFIC ENGINEERED MULTI-PROTOCOL LABEL SWITCHING-TRANSPORT PROFILE (MPLSTP) FOR THE ENHANCEMENT OF QUALITY OF SERVICE." International Journal of Research - Granthaalayah, 5(4) RACEEE, 58-67. https://doi.org/10.5281/zenodo.580626.

\section{Introduction}

To fulfill the huge requirement of data transmission in the current situation, the providers of internet should provide good quality of a service with least possible data drop and time lags. To provide the expected level of reliability and increased level of quality of service, researchers have put in a lot of extra effort for developing mechanisms so that network resources are efficiently utilized and leads to minimized forwarding end-to-end time latency. Multi-Protocol Label Switching technology is one of the mechanisms that brings in the picture of connection- 
Recent Advances in Communication, Electronics \& Electrical Engineering oriented methodology of data transmission in the existing packet switching networks and thus, assures high quality of service guaranties in the traffic flow.

This is a technology that is used as a part of transport of multiple types of traffic such as both the current version of internet protocol and point to point data transfer in any communication transport networks [1]. There has been an exponential growth in the telecommunication sector throughout the world in the past few years, which has led to an incredibly huge amount of traffic being sent from one location to another with different requirements and choices of services. MPLS has proved to be a promising solution that provides different features in the same network of the service provider, thus replacing many transport technologies. The most exceptional feature of MPLS is Traffic Engineering (TE) [1] that allows a service provider to optimize the traffic flow and links utilization as it would be demanded of a service providers network. It gives a substantial amount of control in the hands of the service provider regarding the optimal utilization of the available resources.

There are many reasons why deployment of MPLS has become so popular. The most significant of them is the concept of VPN which segregates the traffic according to the criteria set by the customers, making the connection secure and private. It can be used to establish private connections between different sites of the same customers that might be present at different locations. MPLS also has the capability of protecting its path in case of any failover. This helps the service provider to provide a guaranteed service to its customers. MPLS thus can provide multiple services at the same instant in the same network, justifying its importance in today's networking generation.

MPLS enabled networks are designed for various customers and their various scenarios depending upon the customers' demands in the same network infrastructure. A transparent tunnel can be created between the end points of the network depending upon the class of traffic [2]. All these configurations are done on the service providers end and thus the customer does not have to worry about the routing required or deployment of extra resources.

\section{MPLS: OSI Layer 2.5}

When it comes to communication it is very clear that we first refer to OSI model of seven layers. OSI stands as open systems interconnections model. It is an ideal model that identifies and systemizes the various standard functions of telecommunication system irrespective of the technology that the system is based upon. The different types of communication system that exist today should be able to independently work but still should co-exist in the same communication world using some standard protocols.

There are exactly seven layers of management in the OSI model. An upper layer serves the below layer and thus this lower layer serves its below layer and the chain continues. Open System Interconnection project has provided this seven-layer model in the telecommunication systems.

The project was first demonstrated at the international organization for standardization conference. Since MPLS stands for multi-protocol label switching, and it maps almost all the 
Recent Advances in Communication, Electronics \& Electrical Engineering layer 2 protocols data of open system interface model (OSI), such as Ethernet, SDH, SONET or PDH or ATM. It just maps on to lower layer protocols and gives efficient a transport method. We can say that the MPLS network squeezes in somewhere between layer 2 and layer 3. So, we generally say that it is a layer 2.5 protocol.

OSI Model - 7 Layers

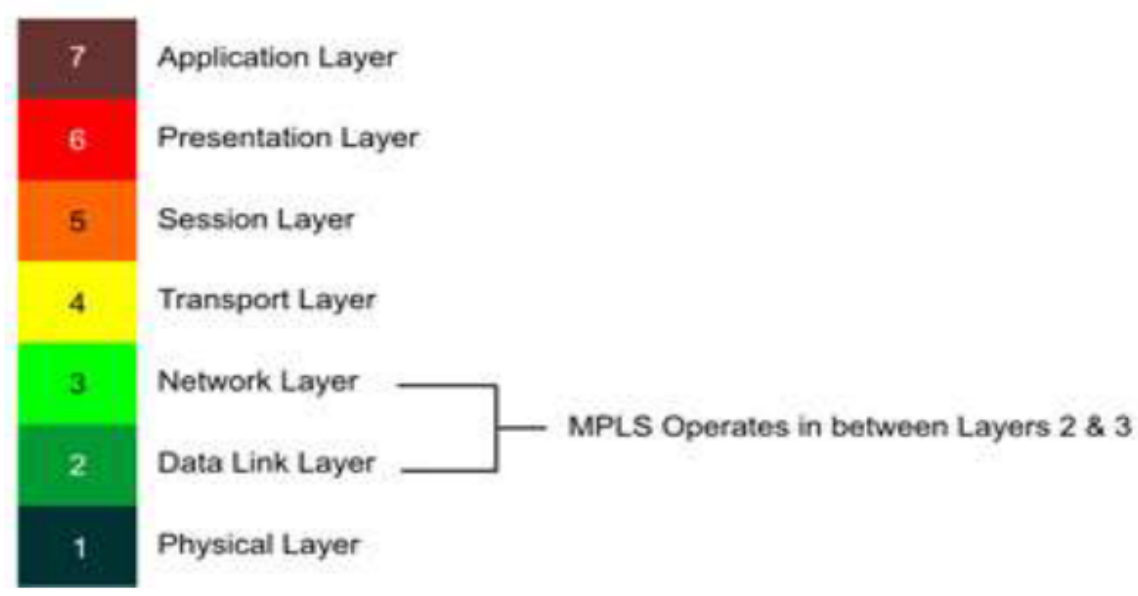

Figure 1: MPLS in OSI model

\section{Traditional Routing and Packet Switching}

Earlier the transport technology was to fulfill the requirements of data transfer like file transfer only. But nowadays the requirements of transport technology are increasing rapidly with more good quality of services like high speed, high bandwidth transmission rates etc.

So, a technology which has a capability to switch level-2 (data link) and level-3 (network layer) was required. The earlier traditional routing and packet switching method helped to provide the need for speed transfer of packets but they did not help to provide service requirements like the information contained in a packet [1]. Almost all traditional technologies were designed for obtaining shortest path in the network for the packet transmission but they did not take care of other quality of services like packet delay, jitter and traffic congestion, which can reduce the performance of network.

Traditional routing protocols used to send the data packets through the same path until the data packets are dropped due to the congestion in the network. Hence there is a clear necessary of utilizing the available network resources efficiently, which leads to low packets delay and packet loss thresholds. The above needs can be mastered using a process called "traffic engineering" and the technology that provides such capability is MPLS.

Usually customers prefer private links for communication between their various offices or anything. But earlier this requested separate links or private links [2] were very costly investment. And again, the customers were not able to use the same provided IP addresses while connecting to the service provider network as the service provider could not able to distinguish between the several customers. Hence a technology which resolves this issue was required. So 
Recent Advances in Communication, Electronics \& Electrical Engineering

ultimately without installing any additional links the customers requires a private or a separate link on same network. This is provided by a MPLS-VPN (Virtual Private Network) technology.

\section{MPLS Label}

The MPLS label, which will be applied on the packets are based on a many of parameters:

- IP network of Destination node

- IP address of Source node

- QoS criterions

- Virtual Private Network (VPN) destination

- Active interface

- Layer-2 circuit

There are two types or forms of MPLS are there, they are as follows,

- Frame Mode MPLS \$-\$ uses a 32-bit label that is infused between

- the Layer-2 and Layer-3 headers.

- Cell Mode MPLS \$-\$ utilized with ATM, and uses the VPI/VCI fields

- ATM header as the label.

This paper will focus on Frame Mode MPLS. The 32-bit mark has the taking after format.

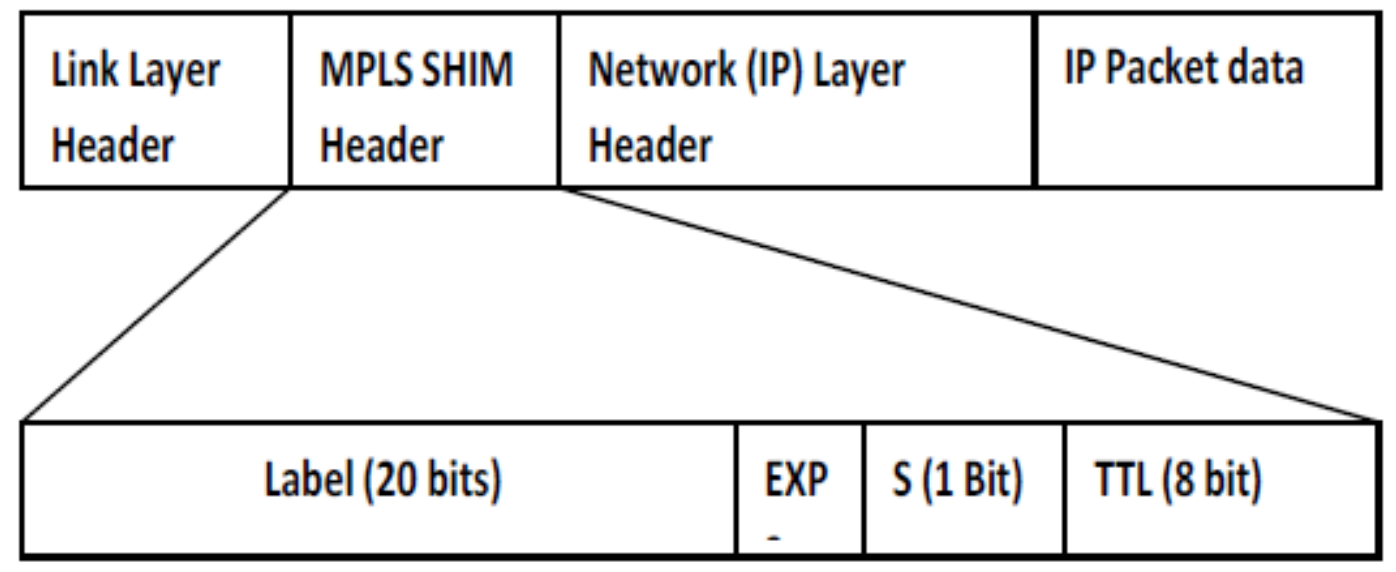

Figure 2: MPLS generic label format.

From the above figure,

- The Label is of 20 bits

- The Experimental is of 3 bits-This field is formally vague, however is utilized by Cisco as an IP priority value [3].

- The Bottom-of-Stack is of 1 bit-This field demonstrates the last label, as various labels are upheld in a similar packet. An estimation of 1 recognizes the last label in the stack.

- The TTL is of 8 bits-This field shows the number of router this label can survive. 
Recent Advances in Communication, Electronics \& Electrical Engineering

An Ethernet header is changed to show the existence of a MPLS label [3].

- 0x8847-shows a labeled unicast IP packet

- 0x8848-shows a labeled multicast IP packet

\section{MPLS Constituents}

- Label: In the MPLS network, the path that is followed is acknowledged by a label and it also permits the routers to enhance routing speed.

- Label switch routing (LSR): LSR sends the packets along label switching path (LSP) depending on the provided label. In receipt of packet LSR checks the lookup table and finds the next hop value [3]. Then it detaches the label from header and attaches new label and then sends to that next hop.

- Label edge router (LER): LER is responsible for adding or removing the labels which are attached to the packets, when they enter or leave MPLS network. So, whenever a packet is entering or leaving MPLS, it should first cross LER router. After this a packet, which enters MPLS via LER is called as "Ingress Router" and a packet which leaves from MPLS via LER is "Egress Router" [3].

- Label distribution protocol (LDP): In this the content of the label will be swapped between the LSRs. Between the routers and the switches, the labels are maintained and also recognized by the LDP.

- Forward equivalence class (FEC): If the set of packets have the similar priority and same behavior then they are forwarded to same path in FEC [3] and these set of packets also have same MPLS label.

- Label switched path (LSP): In the MPLS network signaling protocols sets the path. There will be group of LSPs in MPLS, which get synchronized at ingress router and traverses one or more LSRs and stop egress router. The two routers created LSPs, they are Control driven LSP and explicitly routed LSP [3]. Control driven LSP is set by LDP and is named as hop-by-hop LSP.

\section{Traffic Engineering}

Traffic engineering is a procedure of utilizing the overall available network by trying to uniformly distribute the traffic thought the whole network [4]. Prevention of congestion on any one of the network path is the important result of this traffic engineering procedure. The important feature of traffic engineering is, it does not choose the shortest path between any two devices.

Sometimes it is possible that, during the data flow for any two packets, the packets may follow entirely different path even though the packets have their originating and destination nodes are the same.

By this way the least used network paths can be used and also differentiated services can be proved. The traffic engineering concept is extremely used in the MPLS technology by explicitly using routed paths. 
Recent Advances in Communication, Electronics \& Electrical Engineering

It takes after the guideline of balancing load on different switches, connections and routers to build up the cost productivity and makes the data transmission which is accessible as effortlessly usable. Traffic engineering is utilized with the goal to rouse the stable furthermore, competent IP network operation. Network performance [5] and resource use is enhanced by traffic engineering on the other side. The LSPs are made autonomously, by giving paths which depend on user characterized arrangements. Traffic engineering modifies the routing patterns for keeping away from the effective mapping of network to traffic stream. By this mapping the event of congestion can diminish and can give quality of service (QOS) [6] ensures by playing out a basic part in actualizing network services. The effectiveness of MPLS traffic engineering brings bandwidth reservation, constraint based routing and express routing to MPLS network.

\section{Operation of MPLS}

The source sends its information or data to the destination. In MPLS domain, it's a bit much that all the source traffic is transported through a similar way. On the premise of traffic qualities, distinct LSPs could be made for packets with distinct $\mathrm{CoS}$ fundamentals. The accompanying strides must be taken for data packets to go through MPLS domain.

Step1: - The network powerfully makes directing tables instantly after MPLS empowered router include in inside passage conventions over the network. Label destination protocol (LDP) perform mapping from Label to the destination network [3]. Label destination protocol take the utilization of the routing topologies in the table to set up the label values between the nearby gadgets or devices.

Step2: - The primary intension after a packet enters in entrance (ingress) edge label router is to figure out which layer 3 services it needs, for example, bandwidth administration, (QOS) and furthermore, edge LSR chooses and a label must be applied to the packet header and advances, it.

Step3: - On every packet at first the label value is clarified by the LSR then it is supplanted with new one as per the table and afterward forward the packet.

Step4: - The label value is expelled or removed by Egress edge router then it will read the packet header and after this forward it to the last destination.

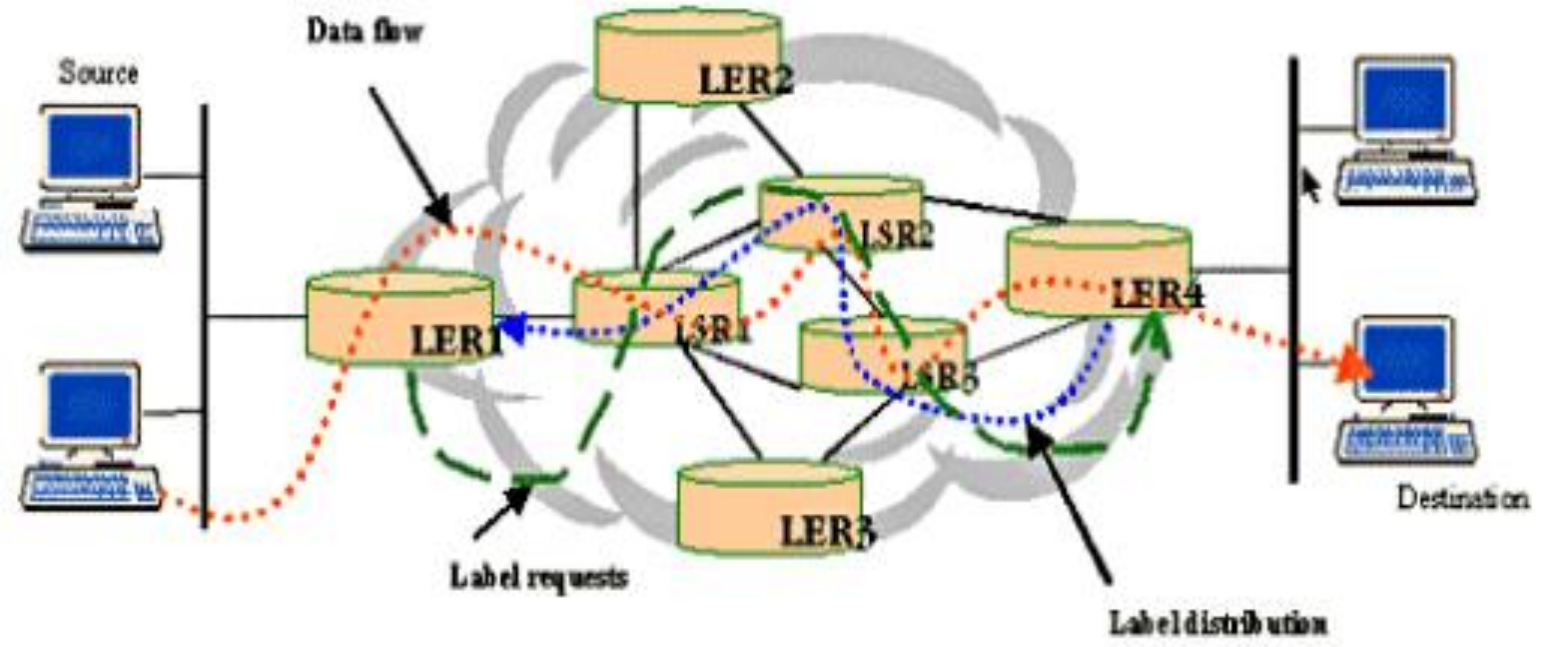

Figure 3: Operation of MPLS 
Recent Advances in Communication, Electronics \& Electrical Engineering

\section{MPLS Tunneling}

As a packet enters in the MPLS network the essential undertaking is to embed a label inside the front of the packet header subsequently the packet is epitomized inside the MPLS network. For labeled packets in the MPLS network makes a label switch path through the network [3]. At that point the packet which goes in the network takes after this name label path instead of routing the packet upheld the goal address inside the IP header. Therefore, tunnel makes adequately through the system by utilizing the PMLS. This tunnel includes all around characterized entrance. The decision of what's permitted into tunnel is overseen by an all-around characterized exit and a door. Into the tunnel packets ought to pass the getting criteria once in light of the fact that inside the tunnel there are no branch exists as the packet isn't directed at transitional mode [7]. Since sheer the system administrator is what will deliver label switched techniques, additionally tunnel entrance can't be create by resentful clients or question the system.

The overhead which is brought about by the MPLS tunneling is rely on the profundity of the label stack. Stream Merging:- MPLS gives the mapping from IP packets to sending proportionality class, at the entrance or ingress to the MPLS space this mapping ought to be performed sheer one time, a sending equality classification could be an arrangement of packet which will be taken care of homogeneously with the point of sending and along these lines it is appropriate for authoritative to a solitary mark frame a sending perspective packet inside steady subset which is dealt with by the label switching router inside a similar linger even through inside the IP header the packet debate from each other with outcome the information [8]. Inside the IP header of the packet the mapping is done between the data and furthermore the sending equality class is many to one. The packet which has exclusively unique substance of their IP header will be mapped into steady sending proportionality classification. For example, an arrangement of packet whose IP goal address coordinates a particular IP address prefix are frequently mapped into a particular sending identicalness class so the steady mark esteem is allocated to the packet range unit take after inside the MPLS space it takes after consistent label switched path. 
Recent Advances in Communication, Electronics \& Electrical Engineering Source

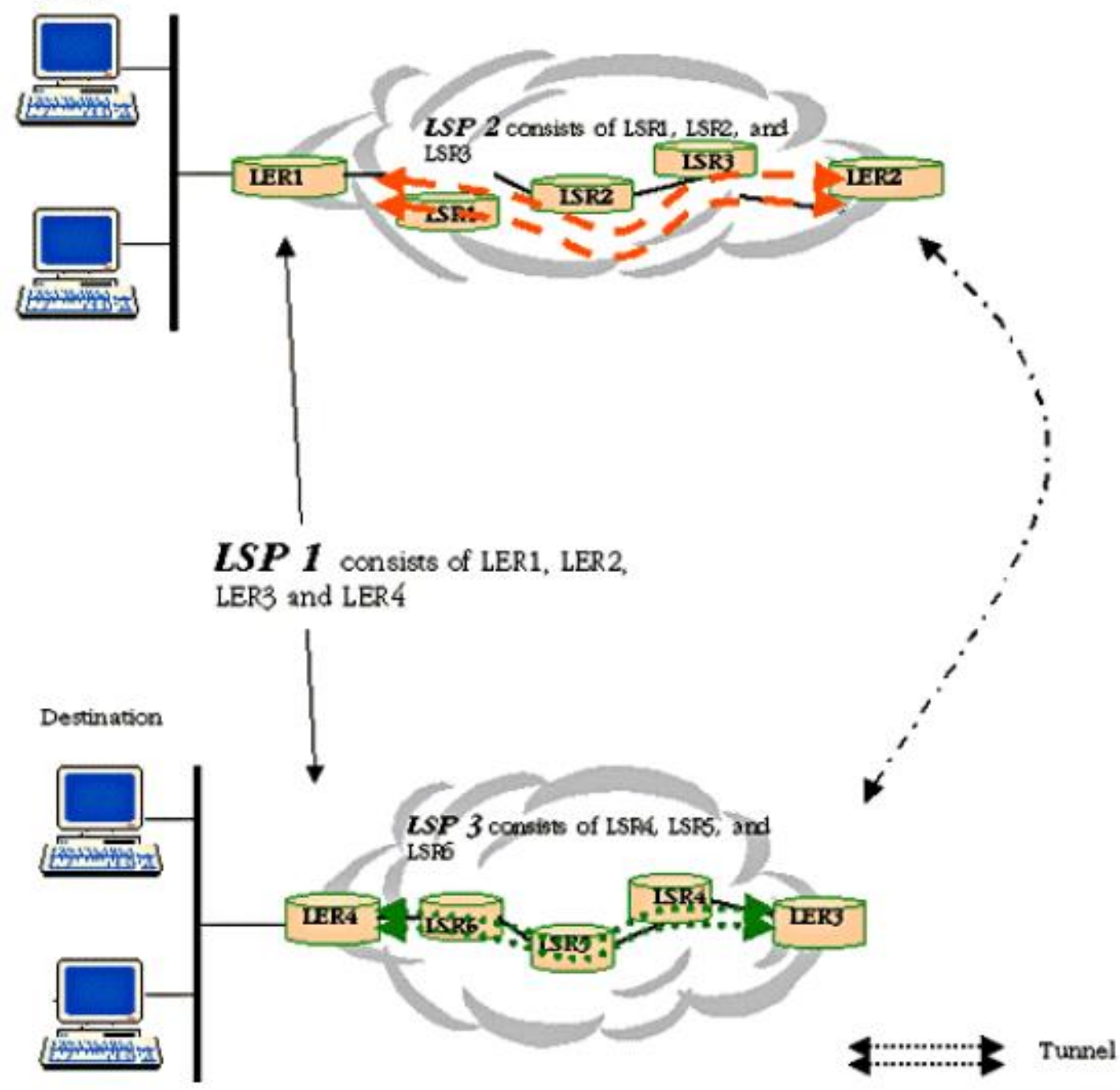

Figure 4: MPLS Tunneling

\section{Drawbacks of Traditional Routing and Packet Switching}

Traditional Routing and Packet Switching had many drawbacks, three drawbacks of traditional IP forwarding, should be highlighted:

- Routing protocols are utilized on all gadgets or devices to disseminate the routing data.

- Despite the Routing protocols, routers constantly forward packets in view of the destination address as it were [9]. The main special case is policy-based directing (PBR) that sidesteps the destination-based directing query.

- Routing queries are performed on each router. Every router in the network settles on a free choice when sending packets.

- MPLS diminishes the number of routing lookups, perhaps changes the sending criteria, and wipes out the need to run a specific routing protocol on every one of the devices. 
Recent Advances in Communication, Electronics \& Electrical Engineering

\section{Applications of MPLS-TP}

MPLS gives numerous spine prerequisites of today's organize. It finishes the accompanying, Enhances packet sending execution in the system

- It improves and disentangles packet sending through routers utilizing Layer-2 switching ideal models.

- MPLS is straightforward, which takes into consideration simple execution.

- MPLS expands organize execution since it empowers routing by switching at wireline speeds.

It bolsters QoS and CoS for service separation

- MPLS utilizes traffic-engineered path setup and accomplishes benefit level assurances.

- MPLS joins arrangements for requirement based and express path setup.

Bolsters systems versatility

- MPLS can be utilized to maintain a $\mathrm{N}^{\wedge} 2$ strategic distance from the overlay issue related with fit IP-ATM networks.

Coordinates IP and ATM in the system

- MPLS gives a scaffold between get to IP and center ATM.

- MPLS can reuse existing router/ATM switch equipment [10], viably joining the two dissimilar networks.

Fabricates interoperable networks

- MPLS is a gauge based arrangement that accomplishes collaboration amongst IP and ATM networks.

- MPLS encourages IP-over-synchronous optical network (SONET) reconciliation in optical switching.

- MPLS assembles adaptable VPNs with traffic engineering ability.

\section{Evolvement of MPLS-TP}

During the last decade though there was lot of improvement in multi-protocol technology, this technology was not sufficient to be practically implemented in the metro load and access entree networks. It was not fast enough to be able to perfectly replace customary transport network. The traditional operators found lot of advantages in this multi-protocol technology. But to adapt this technology, they demanded few suitable changes in IPMPLS. They demanded the exclusion of some of the major features of IP-MPLS [11] such as last but one hop popping, Equi Cost Multiple Paths and Labeling Merge. Thus, it is a new technology with some of the advanced features. This new technology was resilient, with predictability imbibed in it. MPLS-TP came up as an advanced label technology in 2008 after ten years of work on MPLS. 
Recent Advances in Communication, Electronics \& Electrical Engineering

\section{Conclusion}

The real test for MPLS system is to adapt up to the steadily expanding necessities and requests for different services in constrained measure of resources. In the interim it ought to likewise keep up the quality of services as far as packet loss, jitter, delay, protection and security [12]. The insight of routing is joined with the execution of switching by utilizing the Multiprotocol Label Switching (MPLS) and by utilizing an immaculate IP architecture it gives the significant advantages to networks and also with IP and ATM or a blend of other Layer- 2 advancements. This paper illuminated requires actualizing MPLS innovation to conquer a portion of the limits which have confronted in unadulterated traditional IP based forwarding.

\section{References}

[1] Snehal Yadav, Amutha Jeyakumar. "DESIGN OF TRAFFIC ENGINEERED MPLS VPN FOR PROTECTED TRAFFIC USING GNS SIMULATOR." 2016 International Conference on Wireless Communications, Signal Processing and Networking (WiSPNET), Chennai, 2016, pp. 405-409.

[2] E. Rosen, A. Viswanathan and R. Callon. "MULTIPROTOCOL LABEL SWITCHING ARCHITECTURE.” IETF RFC 3031, 2001.

[3] Nisha Chauhan, Vivek Kumar. "A DETAIL REVIEW ON MULTIPROTOCOL LABEL SWITCHING (MPLS)." International Journal of Advanced Research in Computer Engineering \& Technology (IJARCET) Volume 4 Issue 4, April 2015

[4] D. Awduche, J. Malcolm, J. Agogbua, M. O'Dell and J. McManus. "REQUIREMENTS FOR TRAFFIC ENGINEERING OVER MPLS" IETF RFC 2702,1999.

[5] Yan Chen, Toni Farley and Nong Ye. "QOS REQUIREMENTS OF NETWORK APPLICATIONS ON THE INTERNET" International Journal of Information Knowledge Systems Management, 2004, IOS Press (55-76).

[6] Er. Sourabh Jain. July. "PERFORMANCE ANALYSIS OF VOICE OVER MULTIPROTOCOL LABEL SWITCHING COMMUNICATION NETWORKS WITH TRAFFIC ENGINEERING" International Journal of Advanced Research in Computer Science and Software Engineering, Volume 2, Issue 7, 2012

[7] T.Li, Y.Rekhter. "A PROVIDER ARCHITECTURE FOR DIFFERENTIATED SERVICES AND TRAFFIC ENGINEERING (PASTE)” RFC 2430 , Oct. 1998

[8] D.Awduche , J. Malcolm, M. O'Dell, J.McManus, "REQUIREMENTS FOR TRAFFIC ENGINNEERING OVER MPLS” RFC 2702, Sep.1999

[9] J. Chung, "MULTIPLE LSP ROUTING NETWORK SECURITY FOR MPLS NETWORKING", IEEE-MWSCAS, 2002.

[10] S. Alouneh, A. En-Nouaary, and A. Agarwal, MPLS security: an approach for unicast and multicast environments.

[11] Wei Sun Praveen Bhaniramka, Raj Jain "QUALITY OF SERVICE USING TRAFIC ENGINEERING OVER MPLS:AN ANALYSIS".

[12] S.Veni, Dr.G.M.Kadhar Nawaz "A NEW APPROACH TO ENHANCE SECURITY IN MPLS NETWORK"

*Corresponding author.

E-mail address: prabhabiradar50@gmail.com 